\title{
Medulloblastoma with Suprasellar Extension and Atypical
}

\section{Presentation}

\author{
Hilwati Hashim,, ${ }^{1, *}$ Azlindarita Aisyah Mohd Abdullah, ${ }^{2}$ Effat Omar, ${ }^{3,4}$ and Rofiah Ali $^{5}$ \\ ${ }^{1}$ Department of Radiology, Faculty of Medicine, Universiti Teknologi MARA, Malaysia \\ ${ }^{2}$ Department of Opthalmology, Faculty of Medicine, Universiti Teknologi MARA, Malaysia \\ ${ }^{3}$ Centre for Pathology Diagnostic and Research Laboratories, Faculty of Medicine, Universiti Teknologi MARA, Malaysia \\ ${ }^{4}$ Institute for Pathology, Laboratory and Forensic Medicine (iPPerForm), Faculty of Medicine, Universiti Teknologi MARA, Malaysia \\ ${ }^{5}$ Department of Radiology, Hospital Sungai Buloh, Malaysia \\ "Corresponding author: Hilwati Hashim, Department of Radiology, Faculty of Medicine, Universiti Teknologi MARA (UiTM), Sg. Buloh Campus, Jalan Hospital, 4700o Sg. Buloh, \\ Selangor, Malaysia. Tel: +60-123787885, Fax: +60-361265164, E-mail: hilwa167@salam.uitm.edu.my
}

Received 2016 March 18; Revised 2016 November 06; Accepted 2017 January 09.

\begin{abstract}
A 6-year-old boy was referred with symptoms of right partial ptosis, blurred vision and a two-week history of poor coordination and frequent falls. No symptoms of raised intracranial pressure were demonstrated. Imaging showed a large sella mass with extension into the posterior fossa and suprasellar region, suggestive of a craniopharyngioma. Histopathological examination of the postsurgical resection specimen revealed a small blue cell tumor consistent with a medulloblastoma. This case aims to highlight a posterior fossa tumor with an unusual spread and thus clinical presentation.
\end{abstract}

Keywords: Medulloblastoma, Pediatrics

\section{Case Presentation}

A 6-year-old boy was referred to our ophthalmology unit from a district hospital for further investigation on his symptoms of blurred vision and right partial ptosis of two weeks duration. He initially presented to the district hospital following a fall from a staircase. Further history from the child's father revealed that the child seemed uncoordinated. He had fallen several times in the previous two weeks. He dragged his left foot when walking suggesting a left lower limb weakness. He denied any headache or vomiting.

On examination, there was a relative afferent pupillary defect over the right eye with partial ptosis and the eyeball turned outward and downward. There was pallor of the optic disc suggestive of compression of the optic nerve. The left fundus examination was normal.

Extra ocular muscle movement examination of the right eye showed superior, medial, and recti as well as inferior oblique muscle paresis suggestive of a right cranial nerve (CN) III involvement. There was also a right CN VII palsy evidenced by lagopthalmus and facial asymmetry. General neurological examination revealed a left foot drop with the power reduced to $4 / 5$. The reflexes were brisk. All other neurological examinations were normal.

Our working diagnosis at that point was an intracranial bleed causing the third and seventh cranial nerve palsies, as well as the motor deficit (foot drop) secondary to the fall. Intracranial space occupying lesion was also on the list of differential diagnosis.

CT brain performed on the patient showed an illdefined, minimally enhancing suprasellar mass with specks of calcification within. The mass extended to the posterior fossa, causing mass effect onto the brainstem and obstructive hydrocephalus (Figure 1).

The contrasted MRI brain showed an extra-axial lesion at the base of the skull with an epicenter at the sella region. It measured $7.2 \times 4.1 \times 3.7 \mathrm{~cm}$. The lesion extended postero-inferiorly towards the right pons and midbrain compressing these structures as well as the fourth ventricle. However, no mass was identified within the fourth ventricle itself. It also extended superiorly into the suprasellar region invading the cavernous sinus. It was predominantly solid with several intratumoural cysts seen, largest of which was within the suprasellar component. The lesion was hypointense on T1W and slightly hyperintense on T2W and FLAIR and demonstrated mild restricted diffusion. No intratumoural haemorrhage or calcification was seen. Post contrast showed strong enhancement at the sella and suprasellar component but only subtle enhancement in the posterior fossa component. A normal but flattened pituitary gland was identified within the sella. The pineal gland was also preserved. At this point, a differential diagnosis of craniopharyngioma was made, taking into account the suprasellar mass with cystic component. However, it was noted that craniopharyngioma typically does not extend into the posterior fossa (Figures 2 and 3 ). 

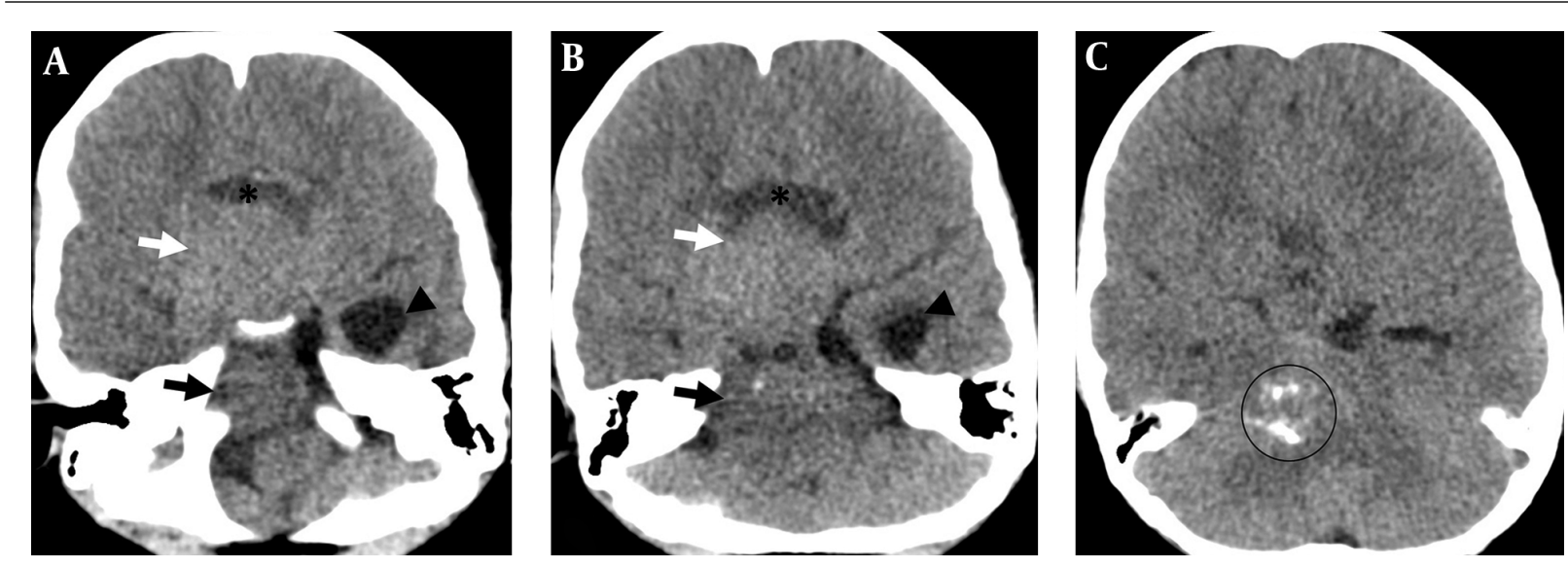

Figure 1. A 6-year-old boy was investigated for symptoms of blurred vision, right partial ptosis and frequent falls of 2 weeks duration. He denied any headache or vomiting. Examination revealed right third and seventh cranial nerve palsies, as well as foot drop. Contrasted axial CT brain at the level of (A) suprasellar and (B) cerebellopontine angle demonstrates an ill-defined, minimally enhancing, solid suprasellar mass (white arrow) with extension into the brainstem (black arrow). It compressed the fourth ventricle resulting in hydrocephalus (seen in this figure as dilatation of the left temporal horn (black arrowhead)). This mass has a cystic component $(*)$. C, Contrasted axial CT brain one slice above image B demonstrates the mass with specks of calcification within (circled).
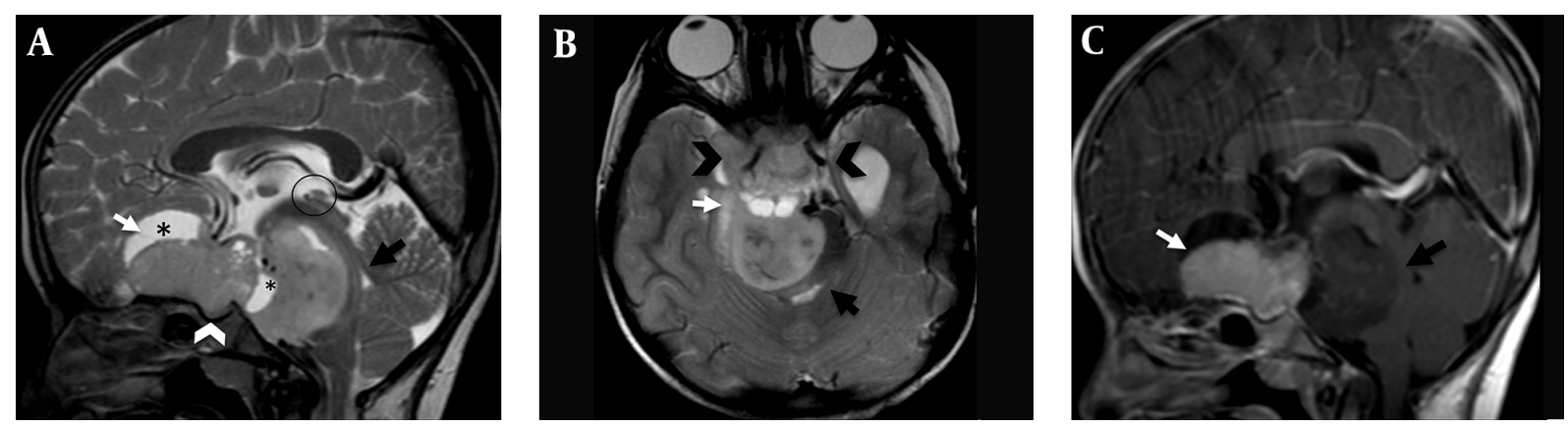

Figure 2. A, MRI of the brain in the same patient in sagittal T2W at the midline shows a lesion at the base of the skull (white arrow) with an epicenter at the sella region (white arrowhead). It is predominantly solid with several intratumoural cysts $\left({ }^{*}\right)$. The lesion extends postero-inferiorly, compressing the fourth ventricle (black arrow). No mass is identified within the fourth ventricle. A, Normal but flattened pituitary gland is identified within the sella (white arrowhead). Pineal gland is also preserved (circled). B, Axial T2W demonstrates the mass (white arrow) extending posteriorly to compress the right pons (black arrow). It also extends antero-superiorly into the suprasellar region, invading the cavernous sinus (black arrowhead). C, Sagittal T1W post contrast demonstrates strong enhancement of the lesion at the sella and suprasellar component (white arrow) but only subtle enhancement in the posterior fossa component (black arrow).

Laboratory investigations showed an elevated prolactin at $533 \mathrm{mU} / \mathrm{L}$ (normal value $59-169 \mathrm{mU} / \mathrm{L}$ ). The rest of the blood investigations and hormonal profile were normal.

A right craniotomy and tumor debulking was carried out. Intra operatively, the right optic nerve was encased with tumor and appeared pale and stretched. The left optic nerve could be partially visualized, but the optic chiasm could not be traced. Anatomies of the sellar and suprasellar structures were distorted with tumor tissue adherent to the frontal skull base dura, right optic nerve, and right internal carotid artery (ICA). Tumor tissues were excised as much as possible, but the tumor that was adhered to the inferior part of the right optic nerve, and right ICA was left behind.
The histopathology examination of the resected tissues revealed a cellular tumor composed of small blue cells with enlarged basophillic nuclei, irregular nuclear membrane, and reduced cytoplasm. The tumor cells formed Homer-Wright rosettes. They were mitotically active and exhibited numerous apoptotic bodies. Anaplasia was not observed. Immunohistochemistry revealed tumor cells positive for chromogranin and synaptophysin. They were negative for gliofibrillary acid protein (GFAP) and cytokeratin (Figure 4). These findings, together with imaging and clinical features led to the diagnosis of medulloblastoma.

Following the histopathology result, the postoperative MRI performed included an MRI of the whole spine that identified drop metastases with extensive syringomyelia (Figure 5). 

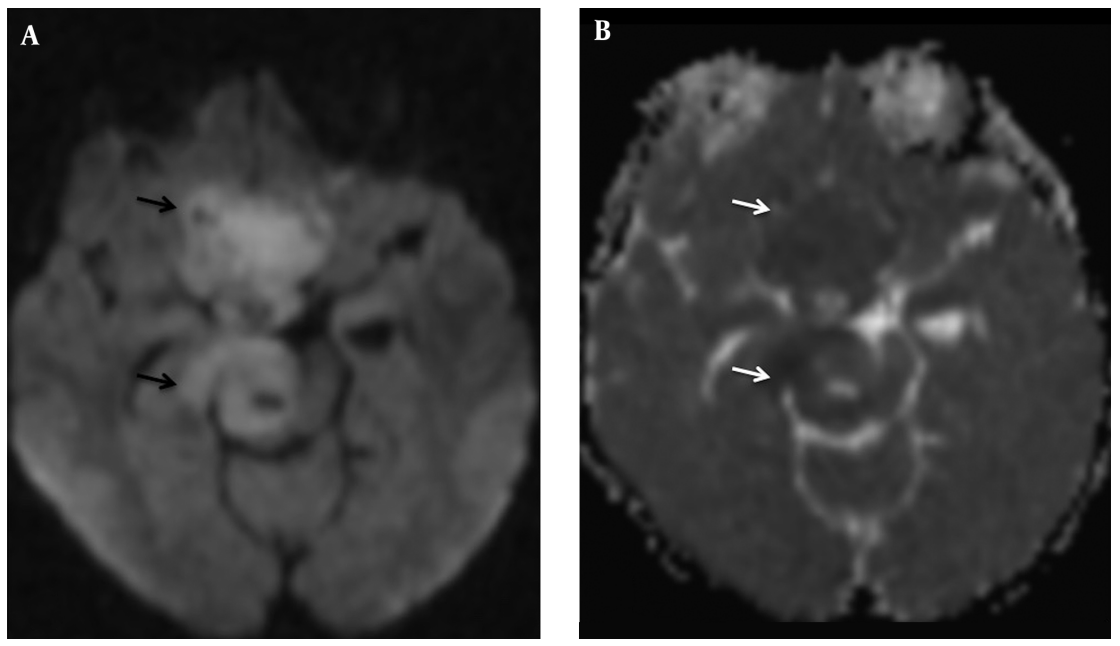

Figure 3. A, Axial diffusion weighted imaging (DWI) demonstrated the tumor to have a mild hyperintensity signal on DWI (black arrow) B, On axial apparent diffusion coefficient (ADC) sequence, the lesion is hypointense (white arrow). These features suggest that the tumor has a mild restricted diffusion.
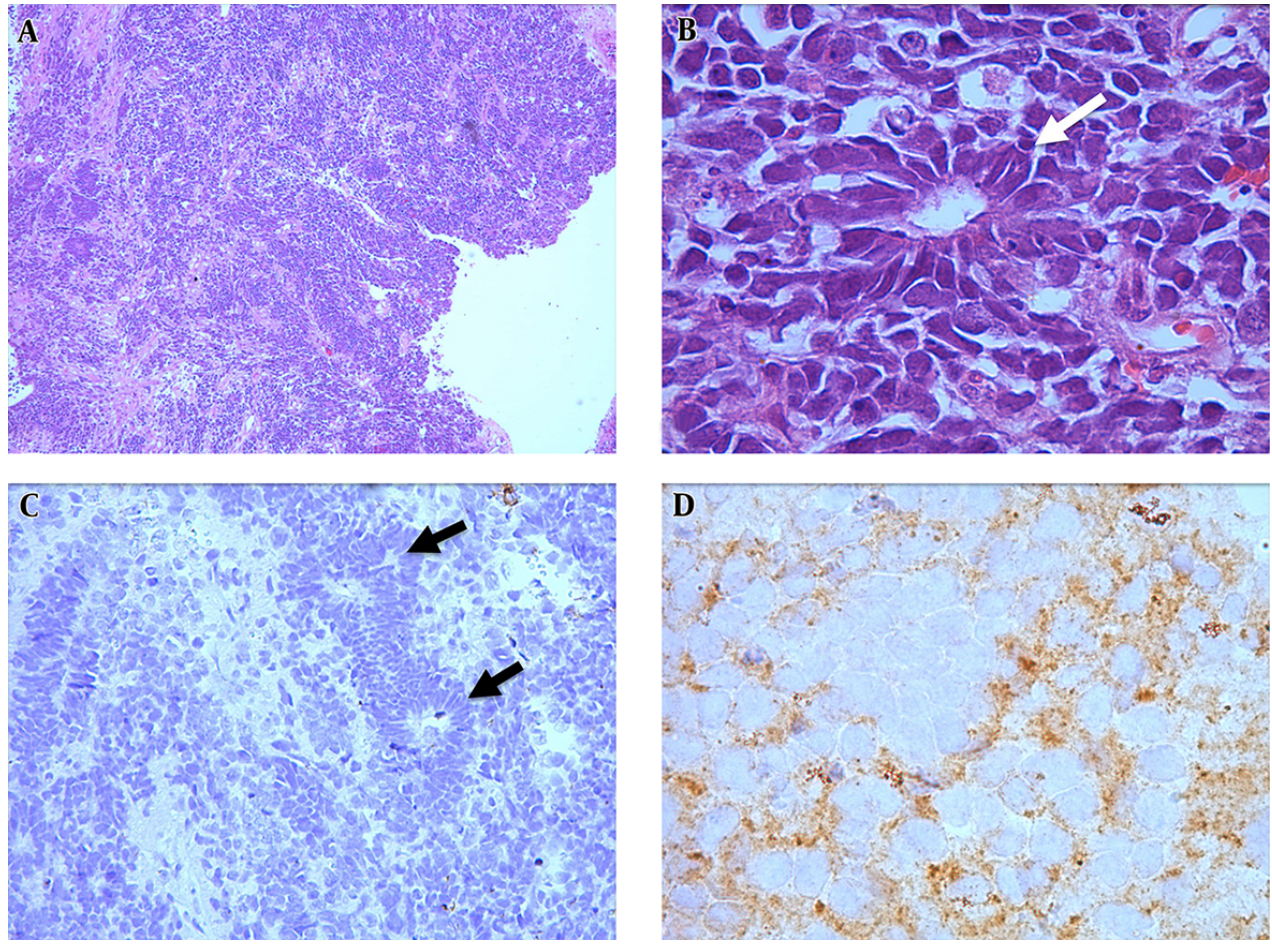

Figure 4. A, Hematoxylin and Eosin, 4X; Tumor fragments show high cellularity; B, Hematoxylin and Eosin, 40X; Tumor cells are small with darkly staining irregular nuclei and form Homer-Wright rosettes (white arrow); C, Glial fibrillary acidic protein (GFAP) stain, 10X; GFAP stain is negative. Two Homer-Wright rosettes are seen in this section (black arrows); D, Chromogranin stain, 40X; chromogranin is positive.

The child was later referred to an oncology unit for radiotherapy. Unfortunately, he succumbed to his illness and passed away on the same day of the transfer.

\section{Discussion}

Medulloblastoma (MB) is the second most common pediatric primary brain tumor (after astrocytoma), account- 


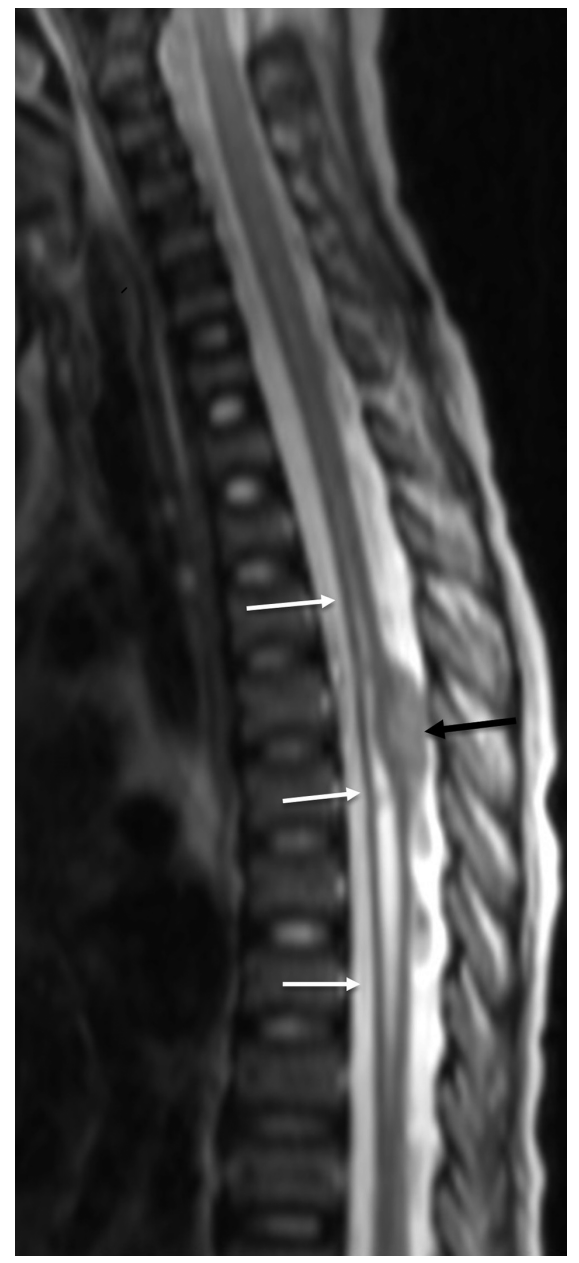

Figure 5. Sagittal T2W of the cervico-thoracic spine demonstrates drop metastases (black arrow) with extensive syringomyelia (white arrows).

ing for $16 \%-18 \%$ of the subtypes $(1,2)$. It is more common in males (M:F 1.2:1) and typically presents between 5 and 7 years of age (1-3). It is one of the embryonal and neuroblastic tumors and was previously known as primitive neuroectodermal tumor (PNET) of the cerebellum.

$\mathrm{MB}$ is typically situated in the posterior fossa (infratentorial). It arises in the midline of the cerebellum and usually obstructs the fourth ventricle resulting in obstructive hydrocephalus $(4,5)$. Thus, the typical clinical presentation is that of raised intracranial pressure (ICP), including headache, and vomiting. It will also cause cerebellar dysfunction resulting in ataxia or gait abnormalities. Our patient did not have symptoms of raised ICP at presentation. What brought him to the hospital initially was falling from the staircase. However, it is important and interesting to note that it was not the fall but rather the symptom of right partial ptosis and blurred vision that prompted a referral to the ophthalmology unit at a tertiary referral center. The ptosis may be explained by the location of the tumor where it compresses the right midbrain tegmentum. The right midbrain tegmentum has the nucleus and fascicles of the oculomotor nerve (cranial nerve III) (6). The tumor also extended beyond the posterior fossa into the cavernous sinus and suprasellar region, possibly compressing the oculomotor nerve as it passed through the cavernous sinus. Further history from the patient's father also revealed a two-week history of poor coordination and frequent falls, which may be attributed to the cerebellar location of the lesion.

Another interesting point about this tumor is its location. Classic medulloblastomas (CMB) are infratentorial and located midline within the fourth ventricle (4). In this patient, the tumor arises from the right pons and midbrain (infratentorial but off midline), and also extends supratentorially into the cavernous sinus and suprasellar region. The fourth ventricle is compressed but is otherwise spared from tumor infiltration. As described above, this attributes to the atypical presentation of the patient. Expansion of the tumor into the supratentorial compartment may be metastatic in nature secondary to cerebrospinal fluid (CSF) dissemination $(4,7)$. This tumor also extends into the right cerebellopontine angle which is rare in MBs (4).

Histologically, MBs can be divided into three subtypes; desmoplastic/nodular MB, classical MB, and anaplastic/large cell MB (high grade) $(4,8,9)$. Classical MB is typically hypointense on T1W and hyperintense on $\mathrm{T} 2 \mathrm{~W}$ with restricted diffusion and variable enhancement (4). Anaplastic MB is associated with increased apparent diffusion coefficient (ADC) value and ring enhancement (10).

In addition, this tumor could also be typed according to four molecular subgroups based on the predominant mutation driving the tumor pathogenesis. They are: Shh, Wnt, Group 3 (having principally MYC mutation), and Group 4 (mutation in isochromosome 17q) MBs (11). The groups have distinct clinicopathological features. The first two groups are better characterized compared to the latter two groups, which is why the latter groups have generic names. Several authors have tried to describe the typical radiological features of the tumor subtypes. Although there is no general consensus yet, most authors described the tumor's location as its predominant feature.

Shh MB arises from granule neuron precursor cells (GNPCs) as a result of an unrestrained Shh pathway. It accounts for $25 \%-30 \%$ of the MBs and has a bimodal age distribution. On imaging, the tumor is typically located in the body of the cerebellum, lateralizing to the sides of the organ $(4,12,13)$. Raleigh et al. also described the tumor as typically isointense on T2-FLAIR sequence (14).

The other three molecular subtypes of MB are typically 
situated in the midline (13). Wnt MB is the smallest subgroup (10\%) and typically arises from the dorsal brainstem and fourth ventricle as a result of activation of the WNT pathway, vital in tumor growth. It could also extend to the cerebello-pontine angle (CPA) (12). Wnt MB has the best prognosis with standard therapy. Group 3 accounts for $25 \%$ of the MBs and has the worst prognosis. It is typically ill defined with ring enhancement and central necrosis. Group 4 medulloblastoma is the largest group (35\%). It typically arises from the fourth ventricle. It is associated with minimal to no enhancement $(4,11,14,15)$.

Our patient had the classical MB type on histopathology, and the tumor is possibly of the Shh molecular subgroup as it arises from the dorsal brain stem rather than the fourth ventricle. Another point to ponder is whether the tumor is an intracranial PNET, arising from the anterior cranial fossa and spreading posteriorly. The immunohistochemical and molecular patterns are similar for both PNET and $\mathrm{MB}$, making differentiating the two an arduous task. However, the almost equal tumor distribution between the suprasellar area and the posterior cranial fossa on imaging studies, and presence of spinal drop metastases supports the diagnosis of MB rather than PNET. Furthermore, MB is the most common childhood intracranial tumor. Fortunately, both tumors respond to the same treatment, making the importance of differentiating them of less urgency compared to if otherwise.

Standard therapy for MBs involve surgical resection followed by adjuvant chemotherapy or craniospinal irradiation. Following the discovery of Shh pathways, targeted therapies utilizing small molecule inhibitors to smoothened (SMO), which is part of the Shh pathway, have been developed. Inhibitors of GNPCs proliferation are also being developed. However, at this point, the prognosis for patients with sonic hedgehog $(\mathrm{SHH}) \mathrm{MBs}$ is still relatively poor. In contrast to SHH MBs, few drugs are developed to target the wingless (WNT) pathway. With standard therapy, Wnt MBs has the best prognosis $(11,15)$.

In conclusion, although $\mathrm{MB}$ is a common pediatric tumor, suprasellar extension of $\mathrm{MB}$ is very rare and may cause atypical presentations.

\section{Acknowledgments}

Department of Neurosurgery and Department of Radiology, Sungai Buloh Hospital; Dr. Wan Azura Yaacob, Consultant Histopathologist, Selayang Hospital, Malaysia. The authors would also like to thank the Director of Health Malaysia for permission to publish this paper.

\section{Footnotes}

Authors' Contributions: None declared.

Financial Disclosure: None.

Funding/Support: None.

\section{References}

1. Rickert $\mathrm{CH}$, Paulus W. Epidemiology of central nervous system tumors in childhood and adolescence based on the new WHO classification. Childs Nerv Syst. 2001;17(9):503-11. doi:10.1007/s003810100496. [PubMed: 11585322].

2. Keene DL, Johnston DL. Pediatric Neuro-oncology: Springer New York; 2015. pp. 9-12.Epidemiology of central nervous system tumors.

3. Maher C, Friedman J. Philadelphia: Lippincott Williams and Wilkins; 2003. p. 985.Posterior fossa tumors in children.

4. Osborn A. Brain: Imaging, pathology, and anatomy. Lippincott Williams and Wilkins Philadelphia; 2012.

5. Sandberg D. Medulloblastomas in children: The international society for pediatric neurosurgery Available from: http://ispn.guide/book/ The ISPN Guide to Pediatric Neurosurgery/Tumors of the Nervous System/Infratentorial Tumors/medulloblastoma-homepage.

6. Ruchalski K, Hathout GM. A medley of midbrain maladies: a brief review of midbrain anatomy and syndromology for radiologists. Radiol Res Pract. 2012;2012:258524. doi: 10.1155/2012/258524. [PubMed: 22693668].

7. Helton KJ, Gajjar A, Hill DA, Boop FA, Kun LE, Langston JW. Medulloblastoma metastatic to the suprasellar region at diagnosis: a report of six cases with clinicopathologic correlation. Pediatr Neurosurg. 2002;37(3):111-7. doi: 10.1159/000064392. [PubMed: 12187055].

8. Louis DN, Ohgaki H, Wiestler OD, Cavenee WK, Burger PC, Jouvet A, et al. The 2007 WHO classification of tumours of the central nervous system. Acta Neuropathol. 2007;114(2):97-109. doi: 10.1007/s00401-0070243-4. [PubMed: 17618441].

9. Eberhart CG, Kepner JL, Goldthwaite PT, Kun LE, Duffner PK, Friedman HS, et al. Histopathologic grading of medulloblastomas: a Pediatric Oncology Group study. Cancer. 2002;94(2):552-60. doi: 10.1002/cncr.10189. [PubMed: 11900240].

10. Yeom KW, Mobley BC, Lober RM, Andre JB, Partap S, Vogel H, et al. Distinctive MRI features of pediatric medulloblastoma subtypes. AJR Am J Roentgenol. 2013;200(4):895-903. doi: 10.2214/AJR.12.9249. [PubMed: 23521467].

11. Taylor MD, Northcott PA, Korshunov A, Remke M, Cho YJ, Clifford SC, et al. Molecular subgroups of medulloblastoma: the current consensus. Acta Neuropathol. 2012;123(4):465-72. doi: 10.1007/s00401-011-0922-z. [PubMed: 22134537].

12. Perreault S, Ramaswamy V, Achrol AS, Chao K, Liu TT, Shih D, et al. MRI surrogates for molecular subgroups of medulloblastoma. AJNR Am J Neuroradiol. 2014;35(7):1263-9. doi: 10.3174/ajnr.A3990. [PubMed: 24831600].

13. Teo WY, Shen J, Su JM, Yu A, Wang J, Chow WY, et al. Implications of tumor location on subtypes of medulloblastoma. Pediatr Blood Cancer. 2013;60(9):1408-10. doi: 10.1002/pbc.24511. [PubMed: 23512859].

14. Raleigh DR, Varenika V, Tihan T, Haas-Kogan DA. Clinical, histopathologic, radiographic and molecular classification of medulloblastoma. JSM Clin Oncol and Res. 2014.

15. DeSouza RM, Jones BR, Lowis SP, Kurian KM. Pediatric medulloblastoma - update on molecular classification driving targeted therapies. Front Oncol. 2014;4:176. doi: 10.3389/fonc.2014.00176. [PubMed: 25101241]. 